\title{
CALCULATION OF THE SURFACE ENERGY OF A FERROMAGNETIC FILM
}

\author{
D. V. Shopova \\ CPCM Laboratory, G. Nadjakov Institute of Solid State Physics \\ Bulgarian Academy of Sciences, BG 1784 Sofia, Bulgaria
}

(Received April 24, 1998)

\begin{abstract}
A new approach to the calculation of the surface energy for a thin ferromagnetic film is proposed. The calculation is done in the mean-field approximation on the basis of the Ising model for temperatures below the bulk transition temperature. In the obtained results the discrete character of the system is preserved in a direction, perpendicular to the surface of the film. The surface energy coefficient is expressed explicitly by the exchange parameters of the system and its temperature dependence is analyzed.
\end{abstract}

Key words: Ising model, mean-field theory, magnetic surface energy, thin ferromagnetic films.

PACS number(s): 75.70.-i, 75.70.Fr

In the last two decades the thin magnetic films and plates have attracted the attention of both experimentalists and theoreticians as the simplest structure for the study of how the surface and finite size effects influence the properties of magnetic systems (see, for example, the reviews in [1] and [2]). Nowadays there are precise ultrahigh vacuum techniques for a controlled growth of magnetic films on a monoatomic level (especially the molecular beam epitaxy) which give an opportunity for the development of the experimental investigation of magnetic structures of reduced dimensions. On the other hand the geometry of thin magnetic films allows the size effects to be taken into account only in one spatial dimension perpendicular to the thickness of the film which extremely simplifies the theoretical description of these systems.

Usually when the magnetic film is thick enough so that the outer layers do not interact directly between themselves, the notion of a surface magnetic energy is used for the theoretical description of the size effects in magnetic systems. This approach has been introduced in the pioneering papers by Kaganov, Omel'yanchuk for a thin ferromagnetic plate [3] and Mills for semi-infinite Heisenberg ferro- and antiferromagnets [4]. They have derived a free energy functional and the form of the surface energy terms with the appropriate boundary conditions for the surface magnetization. The derivation is done in the continuum limit approximation when the thickness of the film is much larger than the bulk correlation length. In the opposite case, when the film consists only of several monoatomic layers it has been shown by Uzunov and Suzuki [5] that within the framework of the Landau mean-field theory, there is a possibility for an analytical description of the thermodynamic properties of the magnetic film. In the intermediate case when the continuum limit cannot be taken in the $z$-direction, usually the properties of the magnetic films are analyzed numerically.

In our paper we shall present a derivation of the surface energy in the intermediate case when the number of the monoatomic layers $n$ is such that the continuum limit does not work along the thickness of the film, i.e when $1<\alpha<n$. As a matter of fact, this is the most frequently met case in the experiments on thin magnetic films and we suppose that our approach will help to explain different experimental situations.

In order to reveal the substantial features of our investigation we shall use a simple model - the Ising model of a ferromagnet taking into account only the nearest neighbour interactions. We consider that the crystal lattice is a simple tetragonal one with basis vectors of the primitive cell $a$ and $c \neq a$. Within this model we can present the thin magnetic film as consisting of $n$ monoatomic ferromagnetic layers in the $z$-direction which coincides with the thickness $L_{z}=L$ of the film, so $L=n c$. The other two spatial dimensions of the film $L_{x}, L_{y}$ are considered large enough such that the size effects do not play any role in the $x y$ plane, which is, therefore, the plane of the ferromagnetic layer. We also suppose that within the layer there is no inhomogeneities like point or plane defects, impurities and any kind of disorder. Thus the inplane exchange $J$ is one and the same for all layers with the exception of the surface layers where it is $J_{s}$. In our idealized model the difference between the surface and bulk layer exchange is due only to the lack of one nearest neighbour for the surface spin in comparison with the bulk spin. Another simplification is that we consider the surface layers to be perfectly clean without the absorbed atoms or any kind of a crystallographic reconstruction in them. This immediately leads to an equal magnitude of the surface magnetizations and a symmetry of the magnetization profile along the thickness of the film with respect to the center of the film. It can be shown that the last condition satisfies the requirement for the minimum of the mean-field free energy and is equivalent to the equality $\sigma(z)=\sigma(-z),(-L / 2) \leq z \leq(L / 2)$ in the continuum limit approximation.

In our calculation of the surface energy we shall preserve the discreteness of the system in the $z$-direction and the layer magnetizations will be denoted by $\sigma_{\alpha}, 1 \leq$ $\alpha \leq n$ and will depend so the layer number $\alpha$. Here we shall introduce two types of boundary conditions for the surface layer magnetizations $\sigma_{1}, \sigma_{n}$ : periodic and free boundary conditions. The periodic boundary conditions in our case are given by the equality $\sigma_{0}=\sigma_{n}, \sigma_{n+1}=\sigma_{1}$. 


\section{V. SHOPOVA}

We should mention here that the introduction of periodic boundary conditions for a confined in the $z$-direction geometry is not a trivial question. This is connected with the fact that the number of the $k_{z}$-vectors in the respective Brillouin zone is discrete and finite. (For more detailed discussion of the periodic boundary conditions, see the original work [5]). The free boundary conditions in our case will be, respectively, $\sigma_{0}=0, \sigma_{n+1}=0$.

Using these definitions for the periodic and free bound- ary conditions, imposed on the surface magnetizations we shall introduce the surface energy as a difference between the mean field free energy densities, calculated, respectively, for the free and periodic boundary conditions [5]. In the Landau expansion of the general mean-field energy density and in the absence of an external magnetic field, the surface energy for the ferromagnetic film will be given by the expression:

$$
\begin{aligned}
g^{f}-g^{p} & =\frac{1}{n} \sum_{\alpha=2}^{n-1}\left\{\frac{1}{2 T}\left(T-z_{2} J\right)\left(\sigma_{\alpha f}^{2}-\sigma_{\alpha p}^{2}\right)+\frac{1}{12}\left(\sigma_{\alpha f}^{4}-\sigma_{\alpha p}^{4}\right)-\right. \\
& \left.-\frac{J_{0}}{2 T}\left[\sigma_{\alpha f}\left(\sigma_{\alpha+1, f}+\sigma_{\alpha-1, f}\right)-\sigma_{\alpha p}\left(\sigma_{\alpha+1, p}+\sigma_{\alpha-1, p}\right)\right]\right\} \\
& +\frac{1}{n}\left\{\frac{1}{2 T}\left[\left(T-z_{2} J_{s}\right)\left(\sigma_{1 f}^{2}+\sigma_{n f}^{2}\right)-\left(T-z_{2} J\right)\left(\sigma_{1 p}^{2}+\sigma_{n p}^{2}\right)\right]\right. \\
& +\frac{1}{12}\left(\sigma_{1 f}^{4}+\sigma_{n f}^{4}-\sigma_{1 p}^{4}-\sigma_{n p}^{4}\right) \\
& \left.-\frac{J_{0}}{2 T}\left[\sigma_{1 f} \sigma_{2 f}+\sigma_{n f} \sigma_{n-1, f}-\left(\sigma_{1 p} \sigma_{2 p}+\sigma_{n p} \sigma_{n-1, p}+2 \sigma_{1 p} \sigma_{n p}\right)\right]\right\}
\end{aligned}
$$

Here $z_{2}$ is the number of the in-layer nearest neighbours, which is supposed to be the same for the bulk and surface layers. $J_{0}$ is the interlayer exchange and in our simple model it is one and the same for all layers but is different from $J$ and $J_{s}$. We have to mention here that a more realistic picture is when the exchange between the surface and the underlying layer is different from $J_{0}$ (see [6]). Our approach gives a possibility also to such a generalization, which can be done in a straightforward way. The subscripts $p$ and $f$ stand for the layer magnetizations for periodic and free boundary conditions, respectively.

It is obvious that for the calculation of the surface energy with the help of expression (1) we need to know the layer magnetizations $\sigma_{\alpha p}$ and $\sigma_{\alpha f}$. It is possible to find them in the general case for arbitrary values of $J, J_{s}$ and intermediate values of $10 \leq n \leq 60 \sim 70$ only numerically. So we have to use the smallness of the parameter:

$$
\frac{\Delta}{z_{2}}=\frac{J_{s}-J}{J_{0}}
$$

in order to find an analytical expression for the surface energy. In our calculations the parameter $\Delta$ is considered positive, which means that the surface exchange is greater than the bulk one; in the opposite case $(\Delta<0)$ there is a possibility for the appearance of an antiferro- magnetic surface layer (see [1]) and we shall not discuss in our paper this case.

We suppose that the layer magnetizations calculated for the periodic boundary conditions are known and are a solution of the appropriate system of mean-field equations. Since the parameter $\Delta / z_{2}$ is small, we can present the layer magnetizations for the free boundary conditions in the following form:

$$
\begin{gathered}
\sigma_{\alpha f}=\sigma_{\alpha p}+\eta_{\alpha}, \\
\eta_{\alpha} \ll \sigma_{\alpha p} .
\end{gathered}
$$

Therefore, we can express the surface energy density from (1) only by the variables $\sigma_{\alpha p}=\sigma_{\alpha}$ and $\eta_{\alpha}$. We shall present the results for the surface energy density for temperatures lower than the bulk transition temperature when the whole magnetic film is ordered. The case of temperatures higher than the bulk transition temperature and the possibility for the appearance of a surface magnetism are thoroughly analyzed in [1], [3] and [4] and we shall not consider them.

Using the fact that the surface layer magnetizations are equal and the ferromagnetic film is symmetric with respect to its center, we obtain the following expression for the surface energy density: 


$$
\begin{aligned}
S= & \frac{1}{2 T n} \sum_{\alpha=2}^{n-1}\left\{\left(T-z_{2} J+T \sigma_{\alpha}^{2}\right) \eta_{\alpha}^{2}-J_{0}\left(\eta_{\alpha+1}+\eta_{\alpha-1}\right) \eta_{\alpha}\right. \\
& \left.+\sigma_{\alpha}\left(\eta_{\alpha+1}+\eta_{\alpha-1}\right)+\eta_{\alpha}\left(\sigma_{\alpha+1}+\sigma_{\alpha-1}\right)\right\} \\
& +\frac{1}{2 T n}\left\{J_{0}(1-\Delta)\left[\sigma_{1}^{2}+\sigma_{n}^{2}+2\left(\sigma_{1} \eta_{1}+\sigma_{n} \eta_{n}\right)\right]\right. \\
& +\left(T-z_{2} J_{s}+T \sigma_{1}^{2}\right) \eta_{1}^{2}+\left(T-z_{2} J_{s}+T \sigma_{n}^{2}\right) \eta_{n}^{2} \\
& \left.-J_{0}\left(\eta_{1} \eta_{2}+\eta_{n} \eta_{n-1}\right)\right\}
\end{aligned}
$$

There is no difference between the magnetizations of the bulk and surface layers when the periodic boundary conditions are applied and only the finite dimension in $z$-direction plays the substantial role. Therefore, we can replace $\sigma_{\alpha}$ by $\sigma$ in the expressions of the type $\left(T-z_{2} J\left(\right.\right.$ or $\left.\left.J_{s}\right)+T \sigma_{\alpha}^{2}\right), 1 \leq \alpha \leq n$, where $\sigma$ is the bulk magnetization.

Then we can easily minimize the expression for the surface energy density (4) with respect to $\eta_{\alpha}$ for the inner layers $2 \leq \alpha \leq(n-1)$ and obtain the following set of equations:

$$
\eta_{\alpha+1}-\frac{T-z_{2} J+T \sigma^{2}}{J_{0}} \eta_{\alpha}+\eta_{\alpha-1}=0 .
$$

The solutions of the above set of finite-difference equations for a symmetric film will be:

$$
\eta_{\alpha}=C \begin{cases}\left(x^{\alpha}-x^{n+1-\alpha}\right) & \text { if } 2 \leq \alpha<(n / 2) \\ \left(x^{n+1-\alpha}-x^{\alpha}\right) & \text { if }(n-1) \geq \alpha>(n / 2) .\end{cases}
$$

Here $C$ is an integration constant which can be found from the free surface boundary conditions and it can be positive or negative depending, as we shall see, on the sign of the difference $(1-\Delta)$. The quantity:

$$
x=\frac{1}{2 d}\left(1-\sqrt{1-4 d^{2}}\right), \text { with } d=\frac{J_{0}}{2\left(T_{c b}-T+J_{0}\right)}
$$

is the smaller root $x<1$ of the characteristic equation of the system $(5) ; T_{c b}$ is the bulk transition temperature and $x \rightarrow 1$ as $T \rightarrow T_{c b}$. It can be seen from (6) that the function $\eta_{\alpha}$ goes to zero at a distance of several layers from the surface, which means that the magnetizations of the inner layers quickly go to the bulk value. Therefore, the influence of the surface is confined to several ferromagnetic layers beneath it.

The quantity $\eta_{\alpha}$ depends both on the temperature and the number of layers which built up the magnetic film, so the temperature dependence of the layer magnetizations will be different from that for the bulk magnetization, $\sigma \sim\left[\left(T_{c b}-T\right) / T_{c b}\right]^{\frac{1}{2}}$. For temperatures close to the bulk transition temperature $\eta_{\alpha}$ goes more slowly to zero in the bulk in comparison with lower temperatures where our approximation works better.

Knowing the solution for the inner layers we can find the surface layer corrections again by a minimization of the surface energy density with respect to $\eta_{1}$ and $\eta_{n}$. The result is following:

$$
J_{0}(1-\Delta) \sigma_{i}+\left(T-z_{2} J_{s}+T \sigma^{2}\right) \eta_{i}= \begin{cases}J_{0} \eta_{i+1} & \text { if } i=1 \\ J_{0} \eta_{i-1} & \text { if } i=n\end{cases}
$$

We cannot solve these equations directly because of the presence of $\eta_{2}$ and $\eta_{n-1}$ in them. We suppose that there is no discontinuity between the magnetizations of the surface and the underlying layer which in our case means that, for example, $\eta_{1}$ goes smoothly to $\eta_{2}$. To a first approximation this can be expressed mathematically by the relation:

$$
\frac{\eta_{\alpha}}{\eta_{\alpha-1}}=x \frac{1-x^{n+1-2 \alpha}}{1-x^{n+2-2 \alpha}} \text {, for } \alpha \ll(n / 2) \text {. }
$$

If the number of ferromagnetic layers $n$ is big enough so that $x^{n+1-2 \alpha} \approx x^{n+2-2 \alpha}$, then we can write, for example, that $\eta_{\alpha} / \eta_{\alpha-1} \approx x, \alpha \ll(n / 2)$. Because of the symmetry of the film with respect to its center, the same is valid also for the surface $n$. Note, that the above considerations lay a restriction on the number of layers, for which our result is valid, i.e. $n \gg 3$. The numerical calculations show that our approximation works well even for $n=10$ at temperatures not too close to the bulk transition temperature, namely, $\left(T_{c b}-T\right) / T_{c b} \geq 0.04$.

Using eq. (9) we can cast the eqs. (8) for $\eta_{1}, \eta_{n}$ in the following form:

$$
\begin{aligned}
& \left(T-z_{2} J_{s}+T \sigma^{2}-J_{0} x\right) \eta_{i}+J_{0}(1-\Delta) \sigma_{i}=0, \\
& i=1, n
\end{aligned}
$$

The solution of eqs. (10) will be:

$$
\eta_{i}=-\frac{x(1-\Delta)}{1-x \Delta} \sigma_{i}, \quad i=1, n
$$

With the help of this expression for the surface layer magnetizations and using the relation $\eta_{\alpha} / \eta_{\alpha-1}, \alpha \ll$ $(n / 2)$, we can find the integration constant in the eqs. (6), which will be:

$$
C=-(1-\Delta) \sigma
$$

Our approximation will be valid, if the following conditions are fulfilled:

$$
\left|\eta_{\alpha}\right| \ll \sigma_{\alpha}, \quad \alpha=1, \ldots, n
$$

which are equivalent to : 


$$
\left|-\frac{x(1-\Delta)}{1-x \Delta}\right| \ll 1,\left|-(1-\Delta)\left(x^{\alpha}-x^{n+1-\alpha}\right)\right| \ll 1 .
$$

The last requirements define the limitations on $n(n>8)$ and give a relation between $x$, i.e. - the temperature and the quantity $\Delta$ - the so-called surface enhancement, which is the measure of the influence of the surface effects on the behaviour of the layer magnetizations in our model. We have shown in fig. 1 the temperature dependence of the surface magnetizations for several values of the parameter $\Delta>1$. It can be seen that for larger values of $\Delta$ and temperatures close to $T_{c b}$ the curve for $\Delta=1.16$ rapidly grows to higher values. This means that the smallness of $\Delta / z_{2}$ is very substantial for the correctness of the calculation.

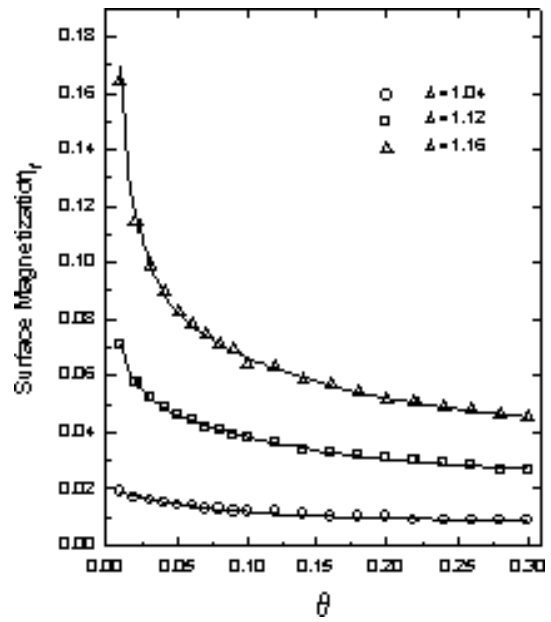

Fig. 1. The temperature dependence $\left(\theta=\left(T_{c b}-T\right) / T_{c b}\right)$ of the surface layer magnetization for $n=12$ layered film and different values of the parameter $\Delta$.

It is obvious from eqs. (6), (11) and (12) that the layer magnetizations depend on the sigh of the quantity:

$$
\lambda^{-1}=1-\Delta
$$

where $\lambda$ is the so-called extrapolation length, introduced by Binder and Hohenberg [7] for magnetic systems. If $\Delta>1$, the surface layer magnetization will be greater than the bulk one and our approximation will be valid when the relation $x<1 /(2 \Delta-1)$ is fulfilled. In the opposite case $\Delta<1$, the surface magnetization is lower than the inner layer magnetizations and the requirement for the validity of our approach gives the relation: $x<1 / \Delta$. The case $\Delta=1$ means an infinite extrapolation length, which is connected with the so-called special transition (for details, see [1]).
The above relations determine the temperature range, for which our approach works well. The numerical calculations show that for $2>\Delta>1,\left(T_{c b}-T\right) / T_{c b}=$ $\theta>0.035$ and for $0<\Delta<1, \theta>0.01$. We show as an illustration the magnetization profile for a different number of ferromagnetic layers in the film and different temperatures in fig. 2 and fig. 3. It can be seen that for temperatures close to the bulk transition temperature the number of layers in the film becomes an important parameter. For comparatively low temperatures the magnetization profile is practically the same, for example, for $n=12$ and $n=30$, (see fig. 2 and fig. 3 ).

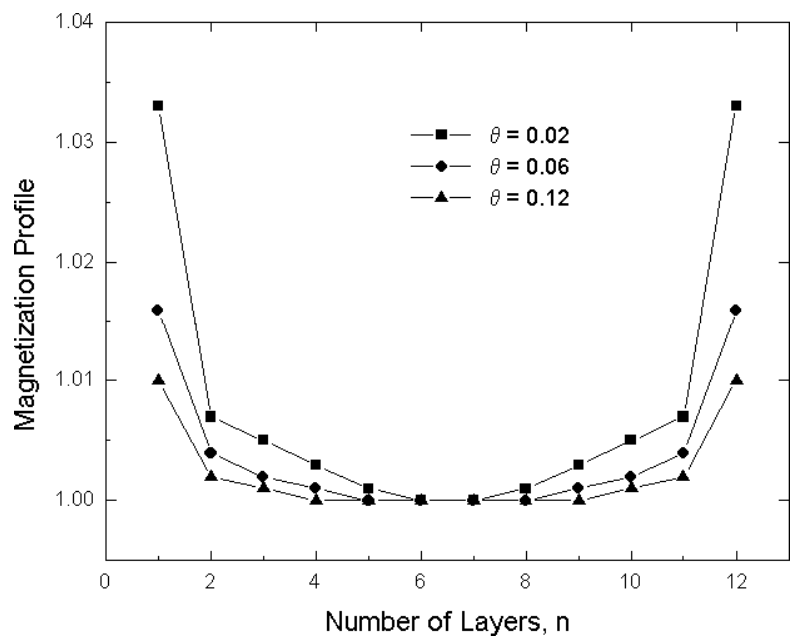

Fig. 2. The magnetization profile $\left(\eta_{\alpha} / \sigma_{\alpha}+1\right), \alpha=1 \ldots n$ along the magnetic film consisting of $n=12$ ferromagnetic layers for three different temperatures and $\Delta=1.04$.

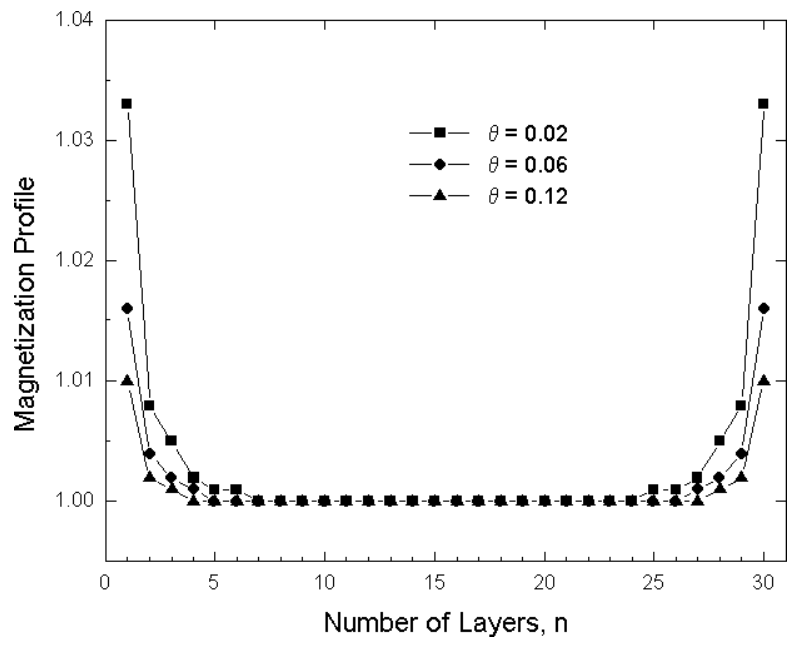

Fig. 3. The magnetization profile $\left(\eta_{\alpha} / \sigma_{\alpha}+1\right), \alpha=1 \ldots n$ along the magnetic film consisting of $n=30$ ferromagnetic layers for three different temperatures and $\Delta=1.04$. 
Now we can replace the solutions for $\eta_{\alpha}, 1 \leq \alpha \leq n$ from eqs. (6) and (11) in the surface energy density eq. (4) and after performing the summation we obtain:

$$
S=\frac{J_{0}}{2 T n}(1-\Delta) \frac{(1-x)}{(1-\Delta x)}\left(\sigma_{1}^{2}+\sigma_{n}^{2}\right)
$$

Note that the surface layer magnetizations in the above expression are calculated for periodic boundary conditions. If we replace $J_{s}$ by $J\left(1+\Delta_{s}\right)$ and $J_{0}=J$, we shall obtain for the extrapolation length $\lambda$ the value $c\left(1-4 \Delta_{s}\right)^{-1}$ which coincides with the value of the extrapolation length obtained in [1] and [3] in the continuum limit approximation.

In the above derived expression for the surface energy (16), there is a temperature dependent factor, which we can include in the definition of the extrapolation length:

$$
\lambda(T, \Delta)=\frac{1-\Delta x(T)}{(1-\Delta)(1-x(T))} .
$$

It can be seen from the above formula that close to $T \sim T_{c b}$, where $x \sim 1$, our approximation does not work. For low temperatures $x \sim d$, see eq. (7) and the temperature factor in $\lambda(T)$ will be of order $(1+\delta(T)(2-\Delta)) /(1+\delta(T))$, where we have denoted by $\delta(T)$ the quantity $\left(T_{c b}-T\right) / 2 J_{0}$.

The temperature dependent extrapolation length determines the temperature dependence of the surface magnetizations. The problem of obtaining the layer magnetizations for periodic boundary conditions is analyzed for the simple case of $J=J_{0}$ in the work [5], but the same method can be straightforwardly generalized for our case, too.
The most substantial feature of our investigation is that in the obtained results the discreteness of the film in the $z$-direction is preserved. We can show that it is not possible to make a straightforward analogy with the continuum limit case. For example, if we apply the free boundary conditions directly to eqs. (3) we shall see that in order to have a complete analogy we should introduce the "fictitious layers" with the indices $\alpha=0$ and $\alpha=n+1$ having magnetizations, respectively, $\sigma_{0}=\Delta \sigma_{1}$ and $\sigma_{n+1}=\Delta \sigma_{n}$, a fact pointed out for the first time in connection with another type of investigation by Pandit and Wortis [8]. Our approach is good only when the surface enhancement is small or can be made such and as we have shown, even in this situation we have to give up the supposition that the layer magnetization varies smoothly with the layer number $\alpha$. Although this approximation works very well in the continuum limit $n>100$, it can be seen that in the discrete case we should go beyond it in order to get a more realistic picture of the properties of the magnetic film.

This means that one should be careful when applying the continuum limit approach to films because the continuum approximation may smear phenomena or characteristic features of the discrete system. The most substantial criterion for the use of the continuum limit approximation is that the respective bulk correlation length should be much greater than the thickness of the film which is different for different substances and experimental situations.

\section{ACKNOWLEDGMENTS}

A support by the NFSR (Sofia) under a contract Ph560 is acknowledged.
[1] K. Binder, in Phase Transitions and Critical Phenomena, vol. 8, edited by C. Domb and J. L. Lebowitz (Academic Press, New York, 1983), p. 1.

[2] S. D. Bader, Proc. IEEE 78, 909 (1990); J. A. C. Bland, B. Heinrich, Ultrathin Magnetic Structures I \& II (Springer, Berlin, 1994).

[3] M. I. Kaganov, A. N. Omel'yanchuk, Sov. Phys. JETP
34, 895 (1972).

[4] D. L. Mills, Phys. Rev. B 3, 3887 (1971).

[5] D. I. Uzunov, M. Suzuki, Physica A 204, 702 (1994).

[6] T. C. Lubensky, M. H. Rubin, Phys. Rev. B 12, 3885 (1975).

[7] K. Binder, P. C. Hohenberg, Phys. Rev. B 6, 3461 (1972).

[8] R. Pandit, M. Wortis, Phys. Rev. B 25, 3226 (1982). 